\title{
Zoo-Housed Chimpanzees Can Spontaneously Use Tool Sets But Perseverate on Previously Successful Tool-Use Methods
}

\author{
Laura M. Bernstein-Kurtycz ${ }^{1,2,3, *}$, Lydia M. Hopper ${ }^{3}$, Stephen R. Ross ${ }^{3}$, and Claudio Tennie ${ }^{3,4}$
}

${ }^{1}$ Cleveland Metroparks Zoo, Cleveland, OH, USA

${ }^{2}$ Case Western Reserve University, Cleveland, OH, USA

${ }^{3}$ Lester E. Fisher Center for the Study and Conservation of Apes, Lincoln Park Zoo, Chicago, IL, USA

${ }^{4}$ Department for Early Prehistory and Quaternary Ecology, Eberhard Karls University Tübingen, Tübingen, Germany

*Corresponding author (Email: lmbk@ clevelandmetroparks.com)

Citation - Bernstein-Kurtycz, L. M., Hopper, L. M., Ross, S. R., \& Tennie, C. (2020). Zoo-housed chimpanzees can spontaneously use tool sets but perseverate on previously successful tool-use methods. Animal Behavior and Cognition, 7(3), 288-309. doi: https://doi.org/10.26451/abc.07.03.03.2020

\begin{abstract}
Tool use is documented in both wild and captive chimpanzees, but the creation of tool sets (e.g., two, or more, tools used in a sequence to solve a task), seems to be less common. This has raised the question of whether tool sets are a culture-dependent trait (CDT), or can be re-innovated independently, and thus fall within chimpanzees' Zone of Latent Solutions (ZLS). To test this, we provided a group of zoo-housed chimpanzees with a novel task that mimicked the wild conditions, required a tool set to solve, and all necessary materials to do so. We therefore enhanced the necessary conditions for the tool-set behavior, but without providing demonstrations of toolset manufacture or use. We baited an artificial termite mound with six ketchup-filled tubes, each with a 45-degree bend and with a cellophane barrier across the top. The chimpanzees needed a rigid tool to pierce the barrier and then a flexible tool to reach the reward. Overall, chimpanzees did use both tool types in order, thus demonstrating that this tool-set form is not a CDT. Yet, few attempts were successful, and the majority were not made using a tool set. This suggests that the behavioral pattern did not stabilize, which may have been due to unintentional difficulty created by the opacity of our task. Overall, our study showed that the form of tool sets can be within the ZLS of chimpanzees, but future studies need to determine what stabilizes the behavioral patterns in the wild.
\end{abstract}

Keywords - Chimpanzee, Tool set, Culture-dependent trait, Zone of Latent Solutions, Innovation

"The wealth of research on tool use and tool construction by chimpanzees and other nonhuman primates has not addressed the fundamental distinction between understanding that tools work versus understanding why they work" (Povinelli, 2000, p. 3).

In the 20 years since the publication of Folk Physics for Apes: The Chimpanzee's Theory of How the World Works, by Daniel Povinelli, much research effort has been dedicated to understanding how, why, and when both wild and captive chimpanzees use tools. Moreover, considerable effort has been expended to reveal how chimpanzees learn (socially or individually) to make, modify, and use tools, and the role of social information on that process. Social learning may also contribute to the coordination of individual learning efforts within a population, namely the socially-facilitated adjustment (including stabilization) of frequencies of individual re-innovations (Tennie et al., in press, 2009, 2020). Special social learning mechanisms can also go beyond mere frequency increases - they can transmit the form of behavior (or artifacts; Tennie et al., in press). It has been suggested that nonhuman great apes, and, in 
particular, chimpanzees, apply such form-transmitting social learning mechanisms, and that they use them in the wild to pass on some of their behavioral forms: passively through imitation (Whiten et al., 1999, 2009) and/or actively, via some variant of teaching (Musgrave et al., 2016, 2020; Sanz et al., 2004). However, the extent to which social learning allows the transmission of a behavioral form, or merely amplifies and harmonizes the frequency of (individually-learned) behavioral forms within populations of wild apes is a topic of some debate (Bandini \& Tennie, 2017; Tennie et al., in press, 2009, 2020; Whiten et al., 2009).

Culture is often defined as "group-typical behavior patterns shared by members of a community that rely on socially learned and transmitted information" (Laland \& Hoppitt, 2003, p. 151). Note that this definition already presupposes that form-copying social learning is required. Therefore, this definition first requires researchers to determine whether a species actually uses form-copying variants of social learning before culture can be ascribed to it. Given that this has not yet been settled in wild apes, a more parsimonious definition of culture is useful, namely one that circumvents the need to identify the exact learning mechanisms involved. Such a definition equates any variant of social learning (form-copying or mere frequency-increasing) with culture. Here, therefore, we adopt the minimal culture definition of Neadle et al. (2017, p. 12): "a behaviour can be considered cultural, if social learning (of any [variant], including learning from artifacts) plays any role at all in the form and/or the frequency of the behaviour (and/or any produced artifacts [...])." Thus defined, culture can be inferred in many animals besides humans (including great apes: McGrew, 1998; van Schaik et al., 2003; Whiten et al., 1999; monkeys: Fragaszy et al., 2004; birds: Weir \& Kacelnik, 2006; whales: Rendell \& Whitehead, 2001; insects: Alem et al., 2016; and fish: Helfman \& Schultz, 1984).

A prerequisite for cumulative cultural forms is the ability to culturally pass on behavioral and/or artifact forms. Humans do this through a variety of mechanisms, including both passive social learning and active teaching (Dean et al., 2012). Humans not only copy the forms of the actions or physical creations of others, but also modify - sometimes unintentionally - and potentially enhance those copied skills and artifacts, which can then again be copied (i.e., cumulative culture; Tomasello, 1996). The upshot of this iterative process is behavioral or technological forms that could not be created by a single, culturally unconnected individual in their lifetime (Boyd \& Richerson, 2005; Mesoudi \& Thornton, 2018; Tomasello et al., 1993). These cumulated forms are also known as culture-dependent traits (CDTs, Reindl et al., 2017). Given that cumulative culture is said to be humankind's niche (Boyd et al., 2011; Pinker, 2010) - and understanding its source in humans has been ranked as one of the top 125 questions of our time (Anonymous, 2005) - understanding the origins and distribution of it has been the focus of extended research efforts.

While apes are certainly capable of various means of social learning (like so many other animals), there is far less evidence that they engage in form-copying social learning, or that they use form-copying in a way that would enable them to build cumulatively on the forms of inventions of others in the way that humans do (Dean et al., 2012; Horner \& Whiten, 2005; Marshall-Pescini \& Whiten, 2008; Tennie et al., in press, 2009). Thus, while human culture is dominated by CDTs, this is not the case for our closestliving relatives, chimpanzees. The upshot is that there may be few, if any, examples of technology forms that chimpanzees use that could not be independently re-innovated by a single individual, i.e., that are beyond chimpanzees' 'Zone of Latent Solutions' (ZLS; Tennie et al., in press, 2009). A "latent solution," therefore, is a behavior and/or artifact form that does not require form-copying social learning mechanisms to occur. These forms are derived individually, although non-copying variants of social learning can regulate and even stabilize latent solution frequencies in populations. Latent solutions, therefore, are the antithesis to form-copying and CDTs (Tennie et al., in press, 2020).

In the wild, chimpanzees use a wide variety of tools (Sanz et al., 2013), yet few, if any, of these tool-use forms may be examples of cumulative cultural learning. Indeed, a number of studies have shown that form-naïve apes (i.e., those not previously exposed to the behavioral and/or tool forms), when provided with the necessary raw materials, spontaneously and individually produced (re-innovated) the same behavioral and tool forms shown by their wild counterparts, thereby demonstrating the lack of need for any form-copying social learning for their production (e.g., Bandini \& Tennie, 2017; Huffman \& 
Hirata, 2004; Menzel et al., 2013; Neadle et al., 2017). Note that these studies are not completely devoid of all variants of social learning effects - by provisioning the subjects with the raw materials, these materials become "enhanced" (an effect that is part of various social learning mechanisms). But enhancement cannot and does not transmit behavioral and/or tool forms (Tennie et al., 2020). These forms can be and are, therefore, re-innovated from scratch by naïve apes - in similar form across diverse populations - meaning that they fall within apes' ZLS. As such, re-innovated behavioral and material forms (i.e., within the ZLS) do not require a form-copying social learning to be acquired - instead, noncopying social learning (including enhancement) likely increases, and then maintains, these form frequencies in those populations (Tennie et al., in press, 2009).

There are, however, a few chimpanzee tool forms that remain candidates for cumulative culture; for example, those that - to human eyes at least - appear to be too complicated to be re-innovatable by naïve chimpanzees. One example is nut-cracking behavior in primates, as it involves several objects used in the correct simultaneous configuration, orientation, and force (capuchins: Corat et al., 2016; Eschar et al., 2016; chimpanzees: Humle, 2002; Luncz et al., 2018). Another example consists of the different "tool set" forms used by some wild groups of chimpanzees. A tool set is defined as the use of more than one type of tool, used in a specific sequential order, to achieve a goal (Brewer \& McGrew, 1990; Sanz et al., 2010). Tool sets are technically cumulative in a loose sense of the term, in that the act involves the sequential combination of at least two tools, but it is unknown if this also means they represent cumulative culture of forms; whether they require form-copying (and thus whether they fall outside chimpanzees' ZLS).

It has been proposed that tool sets can be innovated only when individuals build upon an original foundation of tool use (Sanz \& Morgan, 2009). In other words, the full trait (two tools) may itself depend on the existence of more basic traits first (one tool) and may be developed (perhaps purely individually) only once the basic skill is in place. If this is the case, such tool sets would fulfill at least part of the definition of the ratchet effect (they might then fall into a grey zone of cumulative culture; Tennie et al., in press). However, in spite of the seeming complexity of tool sets, their use has been reported at over 10 sites in the wild (in Cameroon: Deblauwe et al., 2006; Muroyama, 1991; Central African Republic: Fay \& Carroll, 1994; Hicks et al., 2005; Gabon: Boesch et al., 2009; McGrew \& Rogers, 1983; Guinea: Koops et al., 2015; Nigeria: Sommer et al., 2012; Republic of Congo: Bermejo \& Illera, 1999; Nishihara et al.,1995; Sanz \& Morgan, 2007; Sanz et al., 2009; Uganda: Hashimoto et al., 2015) and this relatively high frequency suggests that tool-set behavior might be easier to individually acquire than first thought.

An example of tool-set use can be found among the chimpanzees in the Goualougo Triangle (Republic of Congo). These chimpanzees have demonstrated the use of 22 different tools, and among them, multiple distinct tool sets: one for perforating and dipping at ant nests; one for puncturing and fishing at subterranean termite nests; and one for perforating and fishing at epigeal termite nests; and they also use multiple tools for pounding, lever opening, and fluid dipping at bee hives (Sanz \& Morgan, 2007, 2009; Sanz et al., 2004, 2010). The differences in the two termite extraction tool sets are related to the different types of termite mounds present in this area. To extract termites from an epigeal, or elevated, mound, the chimpanzees first use a perforating tool to open a tunnel through the outside of the mound. For this tool, the chimpanzees use a variety of species of woody plant, usually a twig or short stick, which is near at hand and is procured when needed but is not carried to the mound. After opening a tunnel, the chimpanzee will then insert a fishing tool. These tools are much more specific than the perforating tools; they are long and flexible, and the chimpanzees make them from one of only a few specific species of pliable plant. This form of the tool set differs from that which these chimpanzees use to extract termites from subterranean mounds. For the latter, and in order to open an exit tunnel in a subterranean mound, the chimpanzees use a stout puncturing stick, almost always made of one specific species of plant (Thomandersia hensii), which they often bring with them to the mound, sometimes from several kilometers away. After using sometimes considerable force to open a tunnel, chimpanzees will then use a long, flexible fishing tool (the same as the tools used to fish termites from an elevated mound) to capture termites (Sanz et al., 2004). Because only the resultant tool-set behavior has been observed in the wild, 
not its potential two-step successive innovation, it is unknown whether form-naïve chimpanzees could reinnovate the behavioral and/or artifact form.

In order to test for tool set re-innovation, we extracted the relevant components of the behavioral and artifact forms from wild tool-set behavior. Re-innovation tests can provide subjects with enhancement of the raw materials, as this, in itself, does not necessarily provide information of the behavioral or artifact form (Bandini et al., 2020). Here, we opted to provide subjects with ready-made, stick tools in two variants - rigid and flexible - but we provided no demonstration of the required sequence of their use. In this sense, we wished to determine if task-naïve chimpanzees would spontaneously re-innovate the form of tool-set use when given the "perfect" environmental conditions, even in the absence of a social demonstrator, as none of these chimpanzees had ever been asked to solve this specific problem before and as they had comparable levels of tool experience with single-tool tasks.

We tested this with a group of zoo-housed chimpanzees that had previously acquired and demonstrated proficient one-step tool use in a simulated termite-fishing task using stick tools at an artificial termite mound in their exhibit (e.g., Hopper, Tennie et al., 2015; Lonsdorf et al., 2009). To test our question, we adapted the chimpanzees' artificial termite mound to create a novel tool-use task that required the chimpanzees to use a two-step tool set to obtain food rewards. From the start, we attempted to create conditions as similar to the wild as possible, but there were some differences that could not be overcome, such as the age of the subjects (all adults) and some specifics of the form of the artificial termite mound available to us (see below). Following the model of previous re-innovation tests (e.g., Bandini \& Tennie, 2017; Huffman \& Hirata, 2004; Menzel at al., 2013; Neadle et al., 2017), we provided the chimpanzees with the materials necessary to create the tool set but provided no demonstration of the required solution sequence. The tool set included a rigid tool, needed to pierce a cellophane barrier (step 1), analogous to the puncturing stick used in the wild, and a flexible tool, needed to bend around the curve in an out-of-sight tube to access the food reward (step 2), analogous to the flexible fishing tools used in the wild. Both tools were therefore required and had to be used in a specific sequence (rigid first, flexible second) to obtain the food reward.

For this study we had one key question: would the chimpanzees spontaneously use tool sets? To answer this, we investigated what tools the chimpanzees used at the mound, whether the chimpanzees could successfully pierce the barrier across the tubes to access the food reward, and if they did so by using two different tool types in succession. We also recorded whether they would attempt to modify the tools. We expected that the chimpanzees would spontaneously perform the first step of a tool set by inserting a tool, given that our tested chimpanzees were already familiar with this type of task, and that they would likely attempt to modify the flexible tools. We also expected the chimpanzees to pierce the barrier, given that puncturing behavior appears to fall within the ZLS for chimpanzees (Motes-Rodrigo et al., 2019). Furthermore, if the chimpanzees learned that a rigid tool must precede a flexible tool, we would expect that they would be more likely to use a rigid tool (step 1) before using a flexible tool (step 2), i.e., a tool set. More specifically, and as each hole's barrier needed to be pierced only once in each session, we predicted that the chimpanzees would use rigid tools in every session, but that they would use them when first arriving at the mound and when first dipping at a hole, and thereafter would primarily use flexible tools. Thus, overall, we predicted that the chimpanzees would make a greater number of dips with flexible tools than rigid tools. We also expected that the number of times the food reward was obtained would increase over the course of the study, (and perhaps, plateau eventually), if subjects gained proficiency at the task.

If the chimpanzees successfully used both tools, in order, to retrieve the reward, this would mean that the form of tool-set use falls within the ZLS for chimpanzees. As we predicted that tool-set use is within chimpanzees' ZLS, then we also predicted that the chimpanzees would express this tool-use form spontaneously. If they did not, then this behavior may be an example of a CDT in wild chimpanzees. Furthermore, if the chimpanzees showed the correct order of tool use and if, additionally, the use of a correct order increased and stabilized over time, we would further conclude that even in a somewhat artificial setting and setup and even in a group of adult-only chimpanzees, wild-type group patterns of behavioral use can reappear. 


\section{Method}

\section{Subjects and Housing}

We conducted this study at Lincoln Park Zoo in Chicago IL, USA, with a group of six chimpanzees housed at the Regenstein Center for African Apes. The subjects included two male and four female mother-reared chimpanzees (average age 24.5 years old, range $=15-30$ years). They were housed together in an exhibit comprised of an indoor dayroom $\left(408.4 \mathrm{~m}^{2}\right)$, with both naturalistic and functional elements, including nests, climbing trees, ledges, vines, and mulch substrate, and an outdoor enclosure $\left(1603.3 \mathrm{~m}^{2}\right)$, which could be accessed directly from the indoor dayroom. Within their dayroom was an artificial termite mound (described in "Materials") that these chimpanzees always had access to and all members of the group were proficient at using a single tool to extract food from the mound.

\section{Materials}

We took advantage of the artificial termite mound that was built into the chimpanzees' home exhibit (Figure 1A, see Lonsdorf et al., 2009, for details of its design and construction). The artificial termite mound was designed to be "baited" by the zookeepers from the underside, by attaching PVC tubes filled with a viscous food reward (e.g., ketchup or mustard) to eight points that aligned with holes in the mound's surface through which the chimpanzees could dip for food. Given the design of the termite mound, keepers could bait the mound without having to remove the chimpanzees from the exhibit. Typically (i.e., for enrichment purposes, see below), straight tubes $(4.5 \mathrm{~cm}$ diameter, $33 \mathrm{~cm}$ length) with a food reward in the bottom were attached to the mound, and the chimpanzees could use long sticks as tools to dip through holes on the surface of the termite mound while within their enclosure (Figure 1A).

The artificial termite mound was originally used to study this group's acquisition of tool use and "termite-fishing" (Hopper, Tennie et al., 2015; Lonsdorf et al., 2009). Over the years, the mound has been used to study other questions, including how chimpanzees adjust to changing resource availability and their food preferences in a social context (Bonnie et al., 2012; Calcutt et al., 2014; Finestone et al., 2014). In all of these studies, the tubes used to bait the mound were straight and of uniform length $(33 \mathrm{~cm})$, but the presentation of the task varied in either the number of baited tubes available to the chimpanzees, the days on which baiting occurred, or the reward in the tubes. In addition to its use in experimental studies, the mound was baited regularly as part of the chimpanzees' enrichment schedule. In all previous contexts, the chimpanzees used natural, wood tools that they were either given by keepers or that they had to find by themselves from within their outdoor yard (see Hopper, Tennie et al., 2015 for a detailed description of tool materials with which the chimpanzees had prior experience). We ran our current study during the winter when subjects had minimal access to their outdoor exhibit, and therefore a reduced ability to find their own tools, because we wanted to control the tool types the chimpanzees had access to in test sessions (i.e., the two tool forms we provided).

We modified the setup of the termite mound to require the chimpanzees to use two tools, used in a specific order, to solve the task (i.e., a tool set). We did this by using tubes with a 45-degree bend in the middle (4.5-cm diameter, $69-\mathrm{cm}$ total length) and to which we added a cellophane barrier across the top (Figure 1B). We baited the tubes with a viscous food reward (ketchup) and provided the chimpanzees with two types of novel tools: rigid and flexible. In order to reach the ketchup in the bottom of the tube, the chimpanzees first had to use the rigid tool to pierce the cellophane barrier and then a long, flexible tool, which could bend around the angle in the tube when inserted, to reach the food reward. The rigid tools we provided to the chimpanzees were $0.6 \mathrm{~m}$ long PVC rods $(1 \mathrm{~cm}$ in diameter, Figure $1 \mathrm{C})$ and the flexible tools were $1.2 \mathrm{~m}$ long strips of cardboard $(2 \mathrm{~cm}$ in width, Figure 1C). The long flexible tool had to remain long and unmodified, as if it was shortened too much, it would no longer be long enough to reach the ketchup at the bottom of the tube. 


\section{Figure 1}

The Test Materials we Presented to the Chimpanzees to Assess their Ability to Use a Two-Step Tool Set
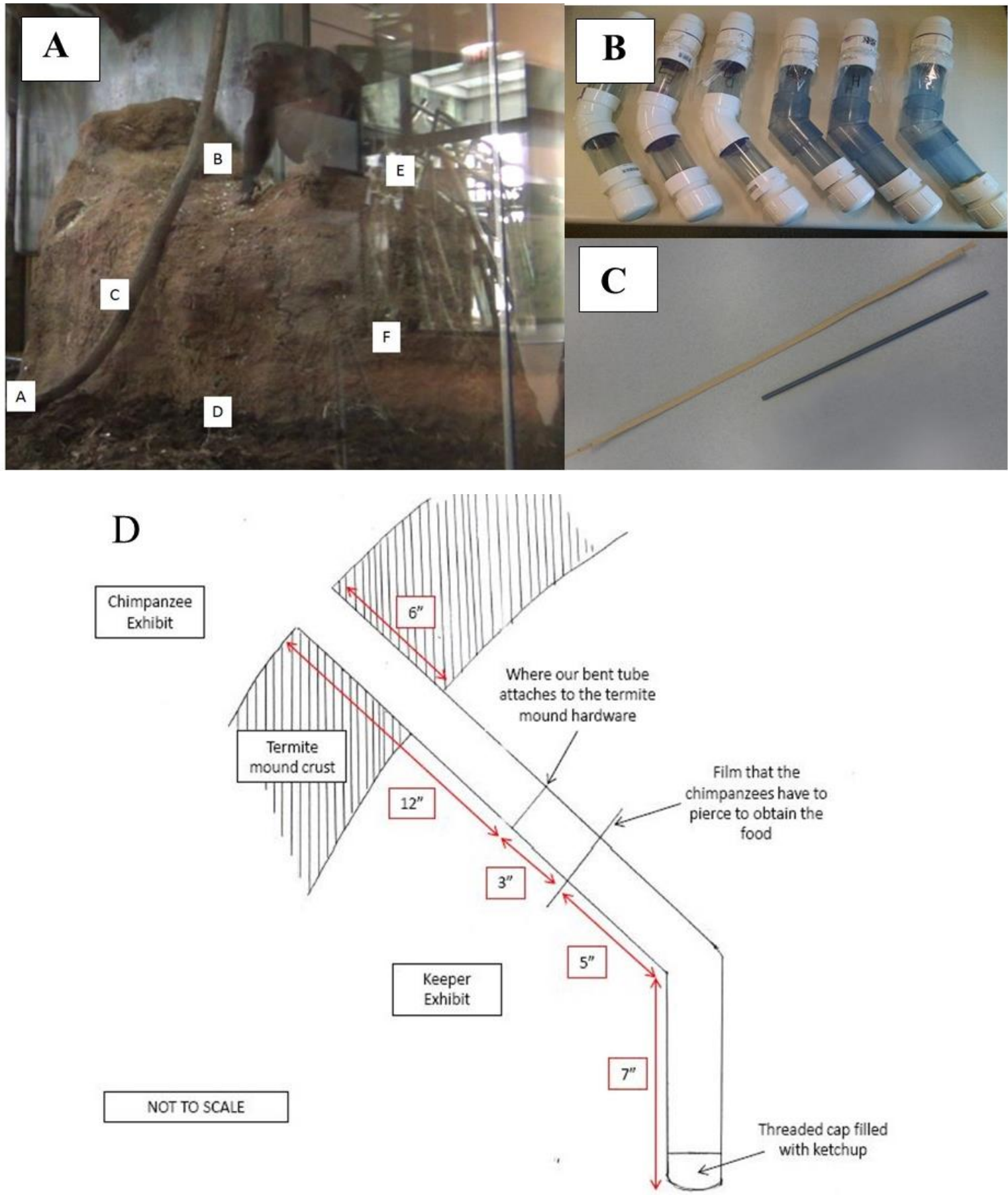

Note. A) The artificial termite mound inside the chimpanzee enclosure. The six holes, A-F, are marked for clarity, B) The six bent tubes with cellophane barrier attached. We placed ketchup in the bottom white cap of the tubes, C) Examples of the flexible cardboard and rigid PVC tools we provided to chimpanzees (one of each pictured), and D) A diagram of the inside of the mound, demonstrating how the tubes were attached. 


\section{Procedure}

We ran ten total sessions, 30 min each, with two sessions per week each starting around $11 \mathrm{am}$. The study lasted five weeks, across February and March 2015. Prior to the beginning of each session, LBK filled the bottom of the six bent tubes with ketchup up to a depth of approximately $5 \mathrm{~cm}$ and covered the tops with a cellophane barrier (a plastic gift bag, unopened, resulting in two layers of stiff cellophane covering the top of the tubes). Each tube was labeled with a letter that matched a labeled hole in the artificial termite mound. Keepers attached the six tubes to the matching holes in the underside of the mound (Figure 1D), and then provided the chimpanzees with 24 tools of each type (48 total) by pushing the tools through a mesh barrier at the perimeter of the chimpanzees' exhibit, $10 \mathrm{~m}$ from the termite mound. This resulted in four tools per type per chimpanzee to ensure that all tools of any one type could not be monopolized by a single chimpanzee. We provided a new set of 48 tools at the start of each subsequent test session but did not provide the chimpanzees with any additional tools during sessions. The termite mound had eight holes in its surface. In the first session, we baited all eight holes, but found that two were difficult to view from the public floor. In the following nine sessions, therefore, we baited the six visible holes, labeled A-F (Figure 1A). At the end of each session, a keeper returned the tubes to LBK, who examined the cellophane barrier and recorded which tubes had been pierced. Using two Sony Handycams (HDR-CX160; Sony, Tokyo, Japan), we recorded the chimpanzees' behavior for 30 min, starting from the moment the tools landed in the exhibit, by filming them at the termite mound from two locations outside the exhibit (see "Coding").

\section{Coding}

The artificial termite mound can be clearly seen from the public floor of the Regenstein Center for African Apes via the floor-to-ceiling windows, allowing for easy viewing of the chimpanzees' behavior within test sessions (Figure 1A). We filmed all sessions from two angles from the public floor and LH and LBK narrated each session during filming as a guide for later coding. After the completion of all ten test sessions, we coded the chimpanzees' behavior from the videos into Microsoft Excel. Inter-rater reliability was conducted using one full, 30-min video, fully coded by both LBK and a volunteer coder (CD), who was naïve to the aims of our study but familiar with this group of chimpanzees. There was high intra-class correlation (ICC; Shrout \& Fliess, 1979) for all measures, including number of dips made with a rigid tool ( $\mathrm{ICC}=.985, p<.001$ ), number of dips made with flexible tools (ICC $=.957, p=.002)$, presence of each individual at or within one meter of the mound when a focal made a dip (Hank (ICC = $.921, p=.010)$, Optimus (ICC $=.973, p=.001)$, Kathy $(\mathrm{ICC}=.955, p=.003)$, Nana $(\mathrm{ICC}=.977, p=$ $.001)$, Cashew $(\mathrm{ICC}=.918, p=.011)$, Chuckie $(\mathrm{ICC}=.978, p<.001)$ ), and number of tool modification behaviors (ICC $=.829, p=.020)$. Following reliability, the rest of the coding was completed by CD.

\section{General Engagement with the Task}

We coded every time a chimpanzee inserted a tool into a hole, inspected the tool, or modified a tool (Table 1), along with the identity of the focal animal performing the action and the identity of any chimpanzees within $1 \mathrm{~m}$ of the focal. Furthermore, for all tool interactions, we coded whether the tool used by the focal was the "rigid" or "flexible" form (Table 1). (While we had aimed to limit the chimpanzees' access to other tool materials during test sessions, occasionally the chimpanzees used other items as tools. Thus, in four sessions, four chimpanzees found sticks within their exhibit that were sharp enough to pierce the cellophane and/or flexible enough to bend in the tubes and reach the ketchup. When the chimpanzees used such sticks, we coded those tools as "other tool.") For each tool insertion behavior ("dip"), we also recorded which specific hole the chimpanzee inserted their tool into. Additionally, for all behaviors, we noted the session number and the time stamp of when the action was performed. 
Table 1

Operational Definitions of Coded Behaviors.

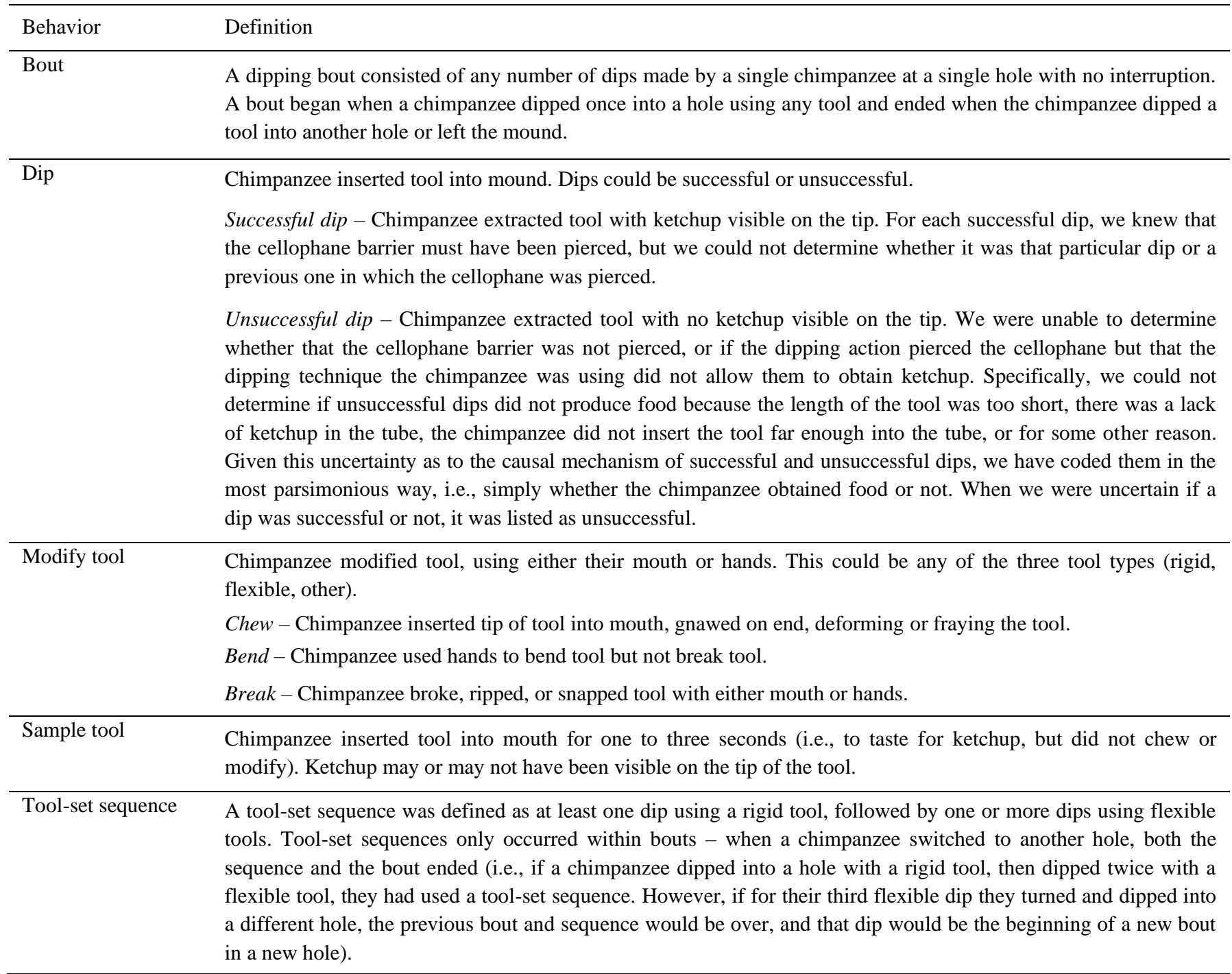

Note. For each of these behaviors, in addition to the described modifiers, we also recorded what type of tool the chimpanzee used (rigid, flexible, or other), the identity of the chimpanzee using the tool, and the identity of any other chimpanzees within $1 \mathrm{~m}$ of the tool using chimpanzee.

\section{Successful Tool Use}

We considered a dip to be successful if the chimpanzee that inserted a tool into a hole was able to procure ketchup with the tool (Table 1). Therefore, each time a chimpanzee completed a dip, we coded whether it was successful or not, irrespective of tool type. Furthermore, we also recorded the total number of holes that the chimpanzees punctured each session. Due to the opacity of the termite mound, we were unable to determine when during the session a given tube was pierced. For each test session, therefore, we coded holes as being successfully pierced based on our examination of the cellophane barrier on each tube at the end of each session (as described in the "Procedure" section above). 


\section{Analysis}

\section{General Considerations and Definitions}

We completed all analyses using R statistical software, version 3.6.1 (R Core Team, 2019). For analysis of the chimpanzees' dipping behavior and potential use of tool-set sequences (see below), we considered each chimpanzee's dipping behavior by bouts. We defined a "bout" to begin when a chimpanzee first dipped a tool (of either type) into a hole and to end when they switched to a different hole or left the mound. Thus, within a single test session, each subject could complete zero, one, or multiple bouts of interaction with the termite mound. Each chimpanzee's bouts were comprised of dips, which were classed as either successful or unsuccessful (see Table 1 for detailed definitions) and could themselves be considered as part of tool-set sequences or not.

Specifically, we defined a tool-set sequence as any time a chimpanzee made a dip with a rigid tool and then made a dip with a flexible tool, one or more times, within a single bout (i.e., in succession at a single hole). We considered any number of flexible dips that followed a rigid dip within a bout to be part of that tool-set sequence, and the sequence ended when the chimpanzee dipped again with a rigid tool or left that hole. Bouts could contain more than one tool-set sequence. Furthermore, successful dips could occur within tool-set sequences and outside of them. For example, if a chimpanzee arrived at a hole and began dipping with a rigid tool one or more times, then picked up a flexible tool and dipped several times and found ketchup on the end of the tool, that successful dip occurred within a tool-set sequence. However, if a chimpanzee arrived at a hole and began dipping with a flexible tool first, and one of those dips was successful, that successful dip occurred outside of a tool-set sequence. This could happen if the barrier at that hole had been previously pierced, or if a chimpanzee was able to pierce a barrier with a flexible tool (which was not intended by design).

\section{General Engagement with the Task}

To assess the chimpanzees' general engagement with the task, we calculated the total number of dips each chimpanzee completed per session, the tools they used for each, and the number of times the chimpanzees modified the tools. To determine if there was a correlation between the chimpanzees' dipping rate and session number (i.e., to evaluate if their dipping proficiency increased with time and if they showed an increased preference for either tool type over time), we used a Pearson's Product-Moment correlation, using the cor.test function, for the number of dips made with both rigid and flexible tools. We also used a Pearson's Product-Moment correlation to see if there were changes in the chimpanzees' rate of tool modification across sessions.

\section{Successful Tool Use}

First, we calculated the total number of dips for each chimpanzee by session and the proportion that were successful. Second, to examine whether the chimpanzees' likelihood of success increased within sessions, we used the cor.test function to correlate chimpanzees' within-session bout number and the number of successful dips they made within that bout (irrespective of whether they constituted a tool-set sequence or not).

We also wanted to explore whether a chimpanzee's success predicted their behavior after the completion of a dip, namely whether they sampled the tool (i.e., put the tool in their mouth) and/or whether they moved to a new hole after a dip. To consider tool sampling rates we compared the best fit of generalized linear mixed models (GLMMs) with "tool type," "session number," "number of observing chimpanzees," "minute in session," and "dip success" as fixed factors; we included "subject ID" and "hole" as random factors in all models. We scored the outcome variable (whether chimpanzees put the tip of the tool in their mouth after a dip or not) as a binary variable. Similarly, to explore what influenced the 
chimpanzees to leave a hole where they were dipping and move to a new hole on the termite mound, we compared the best fit of GLMMs that contained "tool type," "session number," "number of observing chimpanzees," and "dip success" as fixed factors; we included "subject ID" and "hole" as random factors in all models. Again, we scored the outcome variable (whether the chimpanzee moved to a new hole or not) as a binary variable. For both models, given our binary response variables, and the combination of fixed and random predictor variables, we analyzed our data using a binomial generalized linear mixed model. We fit this model using the Laplace approximation method via the 'glmer' function in the 'Ime4' package (Bates et al., 2012). We compared Akaike information criterion (AIC) values to compare model fits and we used Z-tests and likelihood ratio tests (LRTs) to compare the relative contribution of fixed factors to the best model (Bates et al., 2012).

\section{Tool-Set Sequences}

Within each test session, when a chimpanzee first arrived at a hole, they could either use a rigid or flexible tool. As the termite mound was opaque, the chimpanzees could not visually determine when they arrived at a hole for the first time within a session whether the barrier had been pierced. Therefore, the most parsimonious solution, if the chimpanzees adopted the use of tool-set sequences, would be to first dip with a rigid tool. Thus, we used a Pearson's Product-Moment correlation, using the cor.test function, for the proportion of first dips the chimpanzees made with rigid tools across sessions. We also looked at the proportion of dips chimpanzees made with rigid tools for the first dip at each hole $(\max =6)$ within each session. We used a Pearson's Product-Moment correlation, using the cor.test function, for the proportion of first dips at each hole made using a rigid tool.

Given that our definition of a tool-set sequence was for a chimpanzee to dip first with a rigid tool and then to dip one or more times with a flexible tool at the same hole, it is likely that the chimpanzees would use flexible tools more frequently than rigid tools to make dips overall. To determine if chimpanzees chose flexible tools more often than would be expected by chance, we used a Pearson's Chisquare test for count data, using the chisq.test function.

Beyond these inferences about the chimpanzees' tool-set use, we also calculated the total number of (within-bout) tool-set sequences each chimpanzee completed per session.

\section{Results}

\section{General Engagement with the Task}

In total, over the course of all 10 sessions, the chimpanzees dipped a total of 1,728 times. All chimpanzees participated in every session, and all dipped successfully three times or more, with wide individual variation in numbers of dips and bouts (Figure 2, Table 2). The total number of dips that the chimpanzees made over the course of the 10 sessions first increased but then decreased. There was also wide variation in the length of time that each individual engaged with the task (Figure 3). Chimpanzees attempted to modify their tools an average of 49.6 times per session ( $S D: 16.89$, Table 3 ). The two most common modifications included chewing on the end of the flexible tools (37.90\%) and shortening the flexible tools by biting or tearing them to make them shorter $(53.83 \%)$. This second behavior often resulted in flexible tools that were too short to reach to the bottom of the tubes. The frequency of modifications was significantly positively correlated with session number (Pearson's product moment correlation: $r(8)=.67, p=.033$, Table 4). 
Figure 2

Mean Number of Dips Made per Chimpanzee per Session, and Mean Number of Successful Dips Made per Chimpanzee per Session Error Bars Show the Standard Error of the Mean

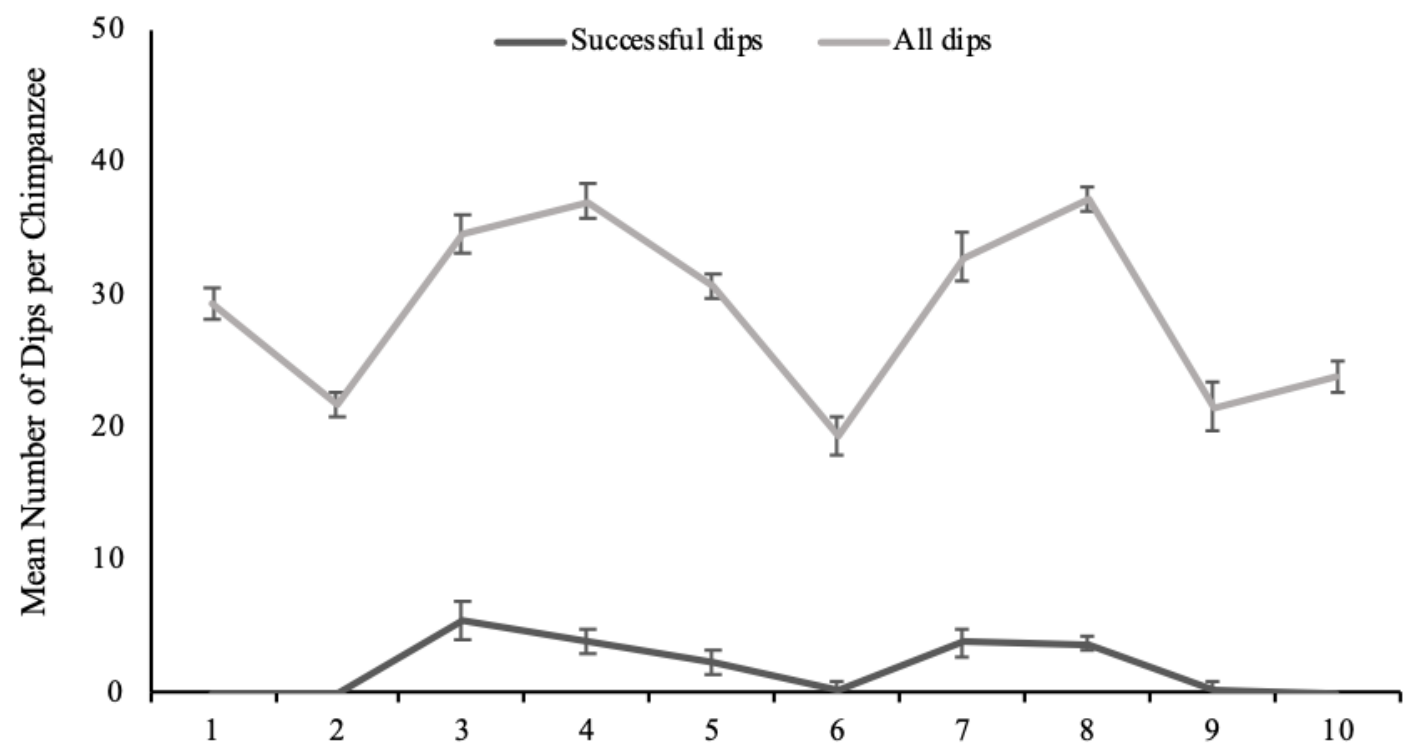

Session Number

Table 2

Individual Variation in Bouts, Sequences, Total Dips, and Success Within Sequences

\begin{tabular}{lcccccccc}
\hline & $\begin{array}{c}\text { Total tool-set } \\
\text { sequences }\end{array}$ & $\begin{array}{c}\text { Proportion of } \\
\text { rigid dips that } \\
\text { led to tool sets }\end{array}$ & $\begin{array}{c}\text { Successful } \\
\text { dips in } \\
\text { sequences }\end{array}$ & $\begin{array}{c}\text { Successful } \\
\text { dips outside } \\
\text { of sequences }\end{array}$ & $\begin{array}{c}\text { Total } \\
\text { dips }\end{array}$ & $\begin{array}{c}\text { Total } \\
\text { bouts }\end{array}$ & $\begin{array}{c}\text { Mean bouts } \\
\text { per session } \\
\text { (SD) }\end{array}$ & $\begin{array}{c}\text { Mean dips } \\
\text { per bout } \\
\text { (SD) }\end{array}$ \\
\hline Cashew & 16 & 0.14 & 12 & 3 & 276 & 76 & $7.6(3.80)$ & $3.75(1.51)$ \\
Chuckie & 31 & 0.23 & 28 & 22 & 517 & 84 & $8.4(3.72)$ & $6.23(1.80)$ \\
Hank & 3 & 0.17 & 0 & 3 & 85 & 31 & $3.1(2.17)$ & $2.98(1.91)$ \\
Kathy & 23 & 0.28 & 3 & 8 & 331 & 76 & $7.6(3.50)$ & $5.04(2.24)$ \\
Nana & 14 & 0.23 & 3 & 35 & 327 & 60 & $6.0(2.28)$ & $6.00(2.26)$ \\
$\begin{array}{l}\text { Optimus } \\
\text { Average }\end{array}$ & 10 & 0.15 & 0 & 2 & 192 & 64 & $6.4(3.35)$ & $3.23(1.23)$ \\
(SD) & $16.17(9.83)$ & $0.29(0.06)$ & $7.67(10.89)$ & $(13.47)$ & $(145.86)$ & $(18.89)$ & $\mathrm{n} / \mathrm{a}$ & $\mathrm{n} / \mathrm{a}$ \\
\hline
\end{tabular}

\section{Successful Tool Use}

In the first seven sessions, the chimpanzees pierced every hole (i.e., every cellophane barrier on each of the six tubes was pierced by the end of the session), but in session 8 , they pierced only five holes and, in sessions 9 and 10, they pierced only 4 holes. Despite their overall success in piercing the holes, the chimpanzees did not extract ketchup from every pierced tube. Collectively, the chimpanzees successfully dipped in three holes in sessions 3, 4, 5, 7, and 8, but successfully from only one hole in sessions 6 and 9 
(in sessions 1, 2, 10, they made no successful dips). Session 6 in general was a low point for dips, bouts, and sequences (Figures 4 and 5).

\section{Figure 3}

Mean Length of Time That Each Chimpanzee Engaged with the Task Within a Session Across all 10 Sessions Error Bars Show the Standard Error of the Mean.

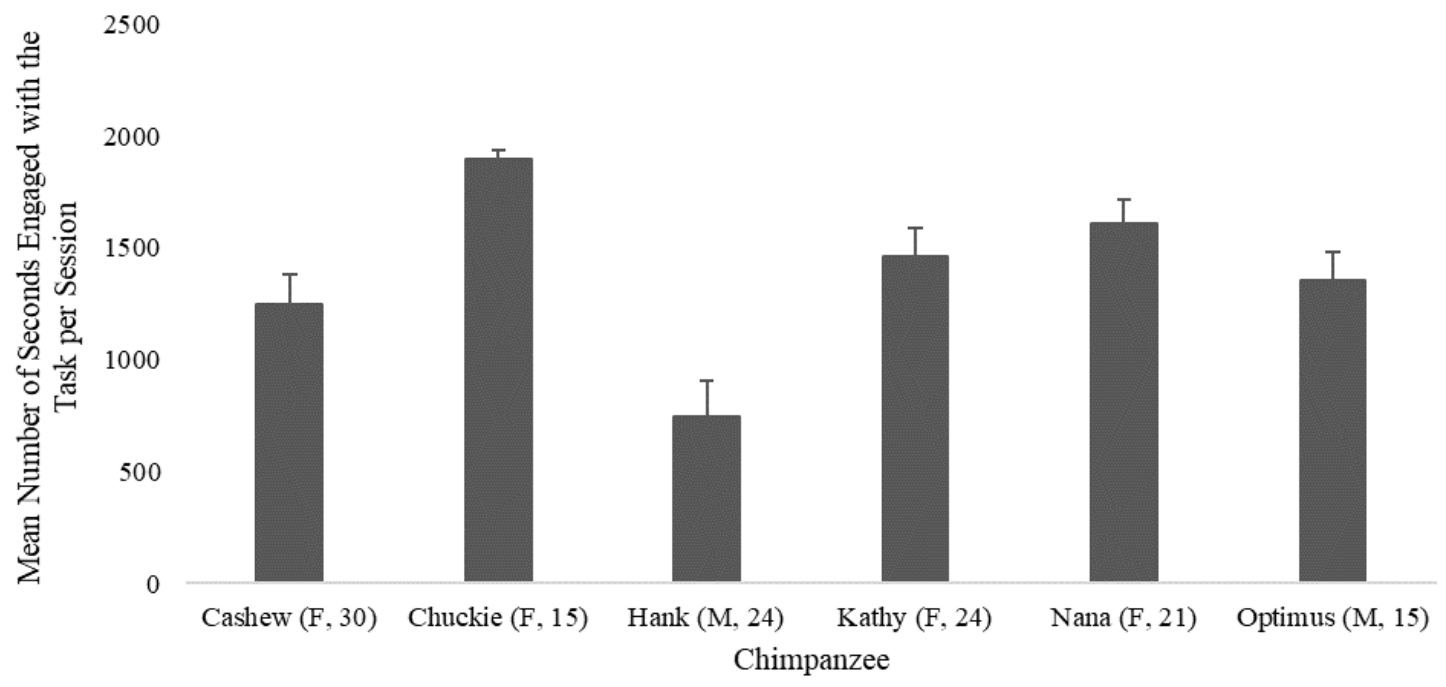

Table 3

All Tool Modification Behaviors Made by the Chimpanzees Across all 10 Sessions

\begin{tabular}{lcr}
$\begin{array}{l}\text { Type of Modification } \\
\text { (followed by tool type) }\end{array}$ & Total count & Percent of total \\
\hline Bend (rigid) & 13 & $2.62 \%$ \\
Bend (flexible) & 1 & $0.20 \%$ \\
Chew (rigid) & 11 & $2.22 \%$ \\
Chew (flexible) & 188 & $37.90 \%$ \\
Chew (other) & 9 & $1.81 \%$ \\
Break with hands (rigid) & 1 & $0.20 \%$ \\
Break with hands (flexible) & 36 & $7.26 \%$ \\
Break with mouth (rigid) & 1 & $0.20 \%$ \\
Break with mouth (flexible) & 231 & $46.57 \%$ \\
Break with mouth (other) & 4 & $0.81 \%$ \\
Other modification (flexible) & 1 & $0.20 \%$ \\
\hline Total & 496 & \\
\hline
\end{tabular}

\section{Table 4}

Number of Modifications Per Session and Frequency of Modifications*

\begin{tabular}{lcc} 
Session & Number of modifications per session & Frequency (number of modifications per minute) \\
\hline 1 & 28 & 0.93 \\
2 & 27 & 0.90 \\
3 & 41 & 1.37 \\
4 & 57 & 1.90 \\
5 & 65 & 2.17 \\
6 & 26 & 0.87 \\
7 & 68 & 2.27 \\
8 & 62 & 2.07 \\
9 & 51 & 1.70 \\
10 & 71 & 2.37 \\
\hline
\end{tabular}

* Number of modifications per session divided by 30 minutes 


\section{Figure 4}

Mean Number of Successful Dips That Occurred Within a Tool-Set Sequence per Chimpanzee per Session and Mean Number of Tool-Set Sequences Made per Chimpanzee per Session Error Bars Show the Standard Error of the Mean

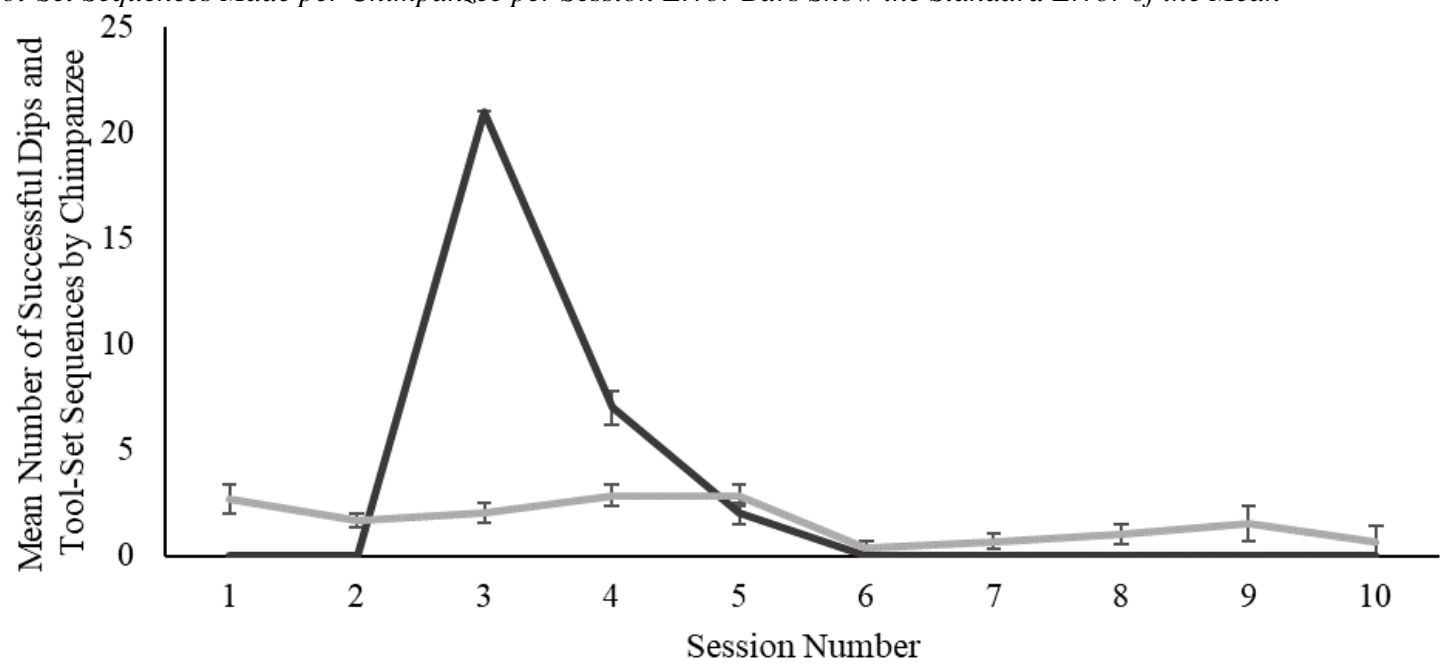

Mean successful dips (within a tool set) _ Mean tool-set sequences

\section{Figure 5}

The Mean Number of Dips (Successful and Unsuccessful Combined) Made with Each Tool Type per Chimpanzee per Session Error Bars show the Standard Error of the Mean

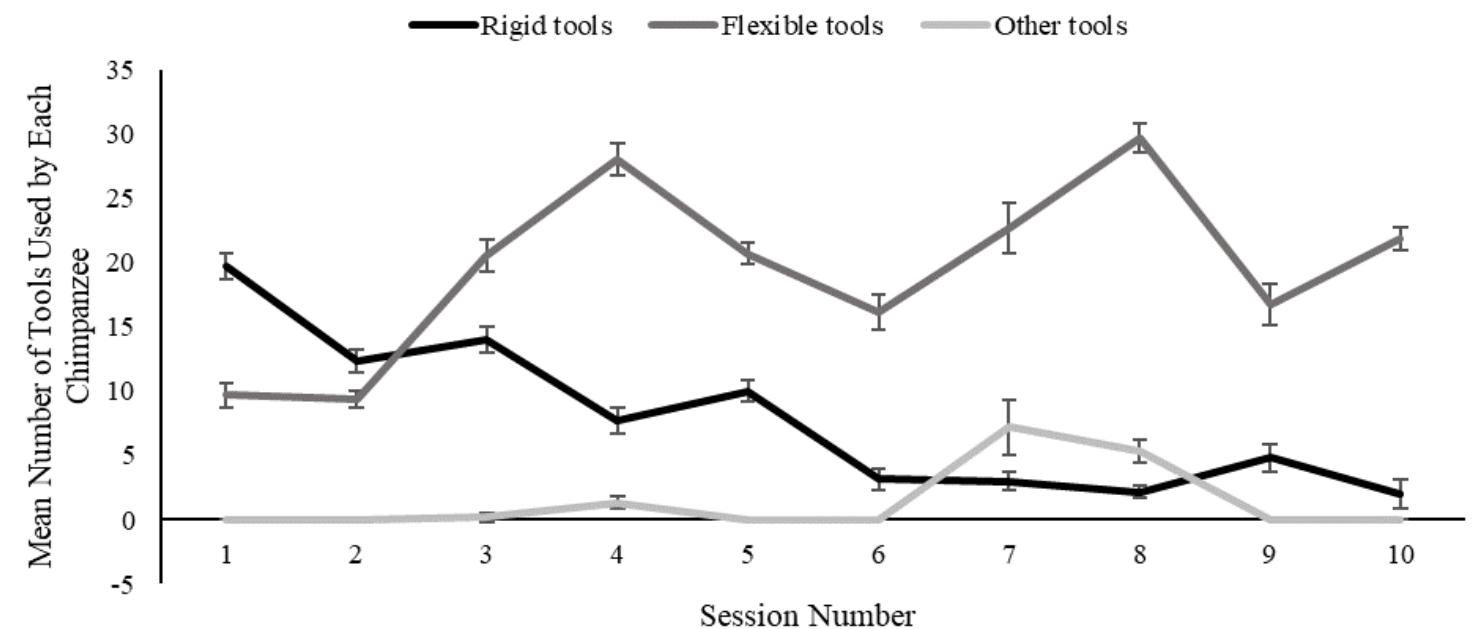

Of the 1,728 dips that the chimpanzees made across all 10 sessions, $119(6.89 \%)$ were successful. All successful dips were made with either a flexible tool $(92.00 \%)$ or an "other" tool $(8.00 \%)$. As intended by our study design, no successful dips were ever made with a rigid tool. Following the chimpanzees' general engagement with the task, the number of successful dips that they made increased over the first few sessions and then decreased, with an average of 1.98 successful dips per session.

Reflecting the variation across chimpanzees in their engagement with the task, there was also individual variation in success; the highest performing chimpanzees dipped the most, used the tool-set sequence the most, and made the most successful dips, though they differed in success rate (Chuckie: 50 successful dips, success rate 9.67\%; and Nana: 38 successful dips, rate 11.62\%). In addition to considering the chimpanzees' success across sessions, we also considered it within sessions and found 
that there was a negative correlation between within-session bout number and number of successful dips the chimpanzees made (Pearson's product moment correlation: $r(390)=-.10, p=.043$ ).

After a chimpanzee had inserted a tool into the mound and extracted it, they did one of four things: they reinserted the tool into the same hole (continuing the bout), they inserted their tool into a different hole (starting a new bout), they sampled the tool, by putting the end into their mouth, and then reinserted the tool back into the same hole (continuing the bout), or they sampled the tool and then inserted their tool into a different hole (ending the bout and starting a new one). The chimpanzees sampled the tool after all (119/119) successful dips but only after 47.23\% (760/1609) of unsuccessful dips. However, the best model that described the chimpanzees' tool-sampling behavior included only "tool type," "session number," and "number of observers" as predictors (Table 5). The rate of the chimpanzees' tool-sampling behavior varied significantly by the type of tool the chimpanzees used (LRT, $\left.\chi^{2}(2)=80.544, p<.001\right)$, the number of observers present for each dip (LRT, $\left.\chi^{2}(1)=6.111, p=.013\right)$, and by session number (LRT, $\chi^{2}(1)=7.015, p=.008$ ). Specifically, chimpanzees were more likely to sample the flexible tool, when more conspecifics were present, and during the later sessions. Considering what predicted the chimpanzees' decision to insert their tool into a new hole after a dip, model comparison revealed that the best model included both "dip success" and "number of observers" as predictors (Table 6). Whether a chimpanzee would switch holes varied significantly by whether the dip was successful (LRT, $\chi^{2}(1)=135.980, p<.001$ ) and the number of observers present as they made the $\operatorname{dip}\left(\operatorname{LRT}, \chi^{2}(1)=6.478, p=.011\right)$. Specifically, we found that chimpanzees were more likely to switch to a new hole if they were not successful on their previous dip and when more conspecifics were present.

Table 5

Mixed Effects Logistic Regression Models and Associated AIC Values, for the Models Predicting Sampling the Tool

\begin{tabular}{lcc}
\hline Factors Included in Model & AIC & Model Rank \\
\hline Tool + Session + Observers & 2239.2 & 1 (best) \\
Tool + Session & 2243.3 & 2 \\
Tool + Observers & 2244.2 & 3 \\
Tool + Minute in session + Session & 2244.8 & 4 \\
Tool & 2250.0 & 5 \\
Tool + Minute in session & 2251.4 & 6 \\
Observers & 2314.5 & 7 \\
Observers + Session & 2315.8 & 9 \\
Minute in session & 2324.2 & 9 \\
Session & 2327.2 & 10 (worst) \\
\hline
\end{tabular}

Table 6

Mixed Effects Logistic Regression Models and Associated AIC Values, for the Models Predicting Switching Holes

\begin{tabular}{lcc}
\hline Factors Included in Model & AIC & Model Rank \\
\hline Success + Observers & 1544.8 & 1 (best) \\
Tool + Success + Observers & 1548.7 & 2 \\
Observers & 1549.3 & 3 \\
Tool + Observers + Session + Success & 1550.3 & 4 \\
Success + Tool & 1550.6 & 5 \\
Observers + Session & 1551.0 & 6 \\
Tool + Observers & 1552.9 & 7 \\
Tool + Observers + Session & 1554.4 & 8 \\
Session & 1677.4 & 9 \\
Tool + Session & 1677.5 & 10 \\
Tool & 1678.3 & 11 \\
Success & 1678.8 & 12 \\
Success + Session & 1678.9 & 13 \\
Success + Tool & 1679.7 & 14 (worst) \\
\hline
\end{tabular}




\section{Tool-Set Sequences}

Chimpanzees demonstrated the correct tool-set sequence (first rigid, then flexible within a single bout) a total of 97 times and an average of $16.17(S D=8.97)$ per session. The proportion of rigid dips that the chimpanzees made to the number of tool-set sequences observed (i.e., the number of times at least one dip with a flexible tool followed a dip with a rigid tool) ranged from $0.14-0.28$ (Table 2). Furthermore, $38.66 \%$ (46) of the total number of successful dips that the chimpanzees made were within tool-set sequences (Table 2), the remainder were made with tools that the chimpanzees dipped into presumably previously pierced holes, which had been pierced earlier in the session by themselves or another group member.

Confirming our predictions associated with tool-set use, a Pearson's Chi-square revealed that chimpanzees chose the flexible tools significantly more often than would be expected due to chance $\left(\chi^{2}\right.$ $(1)=296.350, p<.001)$. Furthermore, rigid tool use decreased over the course of the ten sessions: the number of dips chimpanzees made with rigid tools was significantly negatively correlated with session number (Pearson's product moment correlation: $r(8)=-.81, p=.005$ ). In contrast, flexible tool use increased with time: the number of dips chimpanzees made with flexible tools was significantly positively correlated with session number (Pearson's product moment correlation: $r(8)=.77, p=.009$; Figure 5). On average, the chimpanzees made dips with a rigid tool 47.30 times per session $(S D=34.11)$ and with a flexible tool 117.10 times per session $(S D=38.54)$.

The proportion of first dips the chimpanzees made at a hole using a rigid tool was negatively correlated with session number (Pearson's product moment correlation: $r(58)=-.60, p<.001$ ). A similar pattern was seen within sessions: the proportion of rigid tools used as the first tool by each chimpanzee in a session was negatively correlated with session number (Pearson's product moment correlation: $r(8)=-$ $.83, p=.003$ ). This suggests that, as the study went on, chimpanzees were more likely to use the flexible tools first when arriving at a hole, rather than the rigid tools.

\section{Discussion}

We presented a group of chimpanzees with a novel task requiring a tool set and all the materials necessary to solve the task. We hypothesized that if the ability to re-innovate a tool-set sequence was within chimpanzees' ZLS, then the group would spontaneously re-innovate using tool-set sequences with the materials provided, in a similar manner (i.e., order) to wild chimpanzees. While the chimpanzees were already familiar with using single tools and dipping them into the artificial termite mound in their exhibit to extract food, they were not familiar with the two novel tool types we provided, nor with the need to use two tools in correctly ordered succession to obtain food. When first presented with the task, the chimpanzees immediately began exploring the novel tools and dipping the tools into the mound and all six of the chimpanzees were observed to use a tool-set sequence one or more times. In total, the chimpanzees were recorded to perform a tool-set sequence 97 times, and, on average, a fifth of the times the chimpanzees used a rigid tool, they subsequently dipped with a flexible tool (i.e., used a tool set). More generally, every chimpanzee participated in every session, although participation time decreased in the later sessions and there was wide variation in the number of dips made by the individual chimpanzees. A 15-year-old female (Chuckie) was the most prolific participant and also the youngest, most subordinate member of the group. The fact that she was the most successful, both in participation and in number of successful dips across all sessions and rewards obtained (50 successful dips, representing $42.02 \%$ of all rewards) fits the pattern seen in other zoo studies and in the wild in which young, subordinate individuals have the most reason to innovate, but, perhaps due to their subordinate status, their innovations influence the rest of the group less (Biro et al., 2003; Hopper, Kurtycz et al., 2015; Kendal et al., 2015; Matsuzawa \& Yamakoshi, 1996).

Not only did the chimpanzees engage with the task, but they also successfully extracted food from the termite mound, by piercing the cellophane and then obtaining food using the flexible tools, and 
we recorded nearly 100 instances of tool-set use, with all individuals using a tool set at least one time. From a re-innovation standpoint, one can infer that subjects did innovate the form (Bandini \& Tennie, 2017; Bandini et al., 2020) - here, the sequence of the tool set: first using the rigid tool to pierce the hole and then using a flexible tool one or more times to extract food. Successful dips were slightly more likely to occur earlier within sessions, and rather than a linear relationship between session number and dip success, we saw an increase and then subsequent decrease in the number of successful dips the chimpanzees made over the ten sessions, with no successful dips made in the last session. Additionally, we acknowledge that we observed the chimpanzees successfully puncturing the cellophane and extracting ketchup with a flexible tool (not intended by our experimental design) two times.

However, over the course of the study, not only did we observe a decrease in the number of dips chimpanzees made with rigid tools across sessions (as predicted), we also observed a decrease of rigid tools used at the beginning of sessions and as the first tools used at a hole (counter to our prediction). This may in part be attributed to the fact that the chimpanzees were only ever successful with the flexible tools (by design) and so the use of this tool was reinforced more strongly than were the rigid tools. This pattern could also be attributed to the fact that the chimpanzees could not see the barrier (the barrier was hidden from sight), and thus they could not see the causal purpose for the rigid tool. Taken together, these findings suggest that, while at least one chimpanzee did innovate the tool-set form, the behavior did not stabilize.

While we did not see tool-set use increase or even stabilize over time, all six chimpanzees did use a tool-set sequence and four chimpanzees successfully obtained food using this approach. This shows that the form of this tool-set behavior is principally within chimpanzees' ZLS as we predicted. Therefore, the form of a tool set is not a CDT (sensu Reindl et al., 2017). However, we acknowledge that the frequency of this behavioral form might be under the influence of social learning in the wild (Tennie et al., in press). Given that the frequency of the correct tool order declined over time in our study, there may be some other factor(s) responsible for stabilizing the use of the tools in the correct order over time in the wild that we cannot replicate in a captive setting (see Boesch, 2020, this issue). Indeed, success rates of termite extraction in the wild seem to be higher than we saw here for liquid extraction (McGrew \& Marchant, 1999). However, as in the wild, a number of social and asocial factors may influence the expression of a behavior, and next we consider the elements that may have influenced, or inhibited, the expression of the tool-set form in the group of chimpanzees we studied here.

\section{Individual Learning and the Social Environment}

Our study was conducted with the chimpanzees in a social setting, mimicking wild conditions. However, once the first chimpanzee had re-innovated the use of a tool set, we cannot be certain whether subsequent individuals to do so were primed by their observations of them, in this case, Chuckie in session 1 (sensu Fiore et al., 2020). Indeed, the ZLS hypothesis predicts that non-copying forms of social learning likely influence the frequency at which latent solutions are expressed. Yet, we did not see toolset use become habitual. We argue that an absence of (trained) social models is unlikely to be the missing factor in our study as to why tool set use did not become habitual. Indeed, the use of the correct tool order did appear - in each tested individual - and resulted in success, creating a potential source of social learning for others. Yet the correct order, while present, was not often realized, and most of the individuals largely abandoned the activity by the later sessions, suggesting that they did not have a causal understanding of the task.

With regard to individual learning, our task might have been less naturally structured, or guided, than may be the case the wild. Although we created a task that required both tools in correct order to be solved - our task did not require both tools and this correct order once it was partly solved. After initial piercing and dipping, rewards were left in the task that remained sufficient to be exploited. This is clear from the fact that many successful dips were made at previously pierced locations by subjects other than the initial piercer - without the need to use the entire tool set anymore. This was an outcome of the type of artificial termite mound setting available to us, where the contained viscous reward was a long-term 
exploitable resource. That is, our task may not compare in this respect well to the wild, where a pierced hole may be (at least temporarily) exploited beyond a rewarding level - guiding the production of further pierced holes and discouraging the re-use of others' pierced holes.

In the wild, feeding at termite mounds is often solitary or performed in mother-offspring dyads (Lonsdorf, 2006). We tested the chimpanzees in a group setting, which changes the learning environment and the chimpanzees' access to the resources (both the mound and the tools). Indeed, in our study we found that a higher number of conspecifics present on the mound contributed to the likelihood of an individual switching to a new hole and the likelihood of an individual sampling the tool. Whereas this group has previously shown the ability to take turns when accessing a reduced number of baited holes at the termite mound (Calcutt et al., 2014), it appears that when more holes are baited, and they have the option to switch holes, this is influenced by the number of conspecifics near them. The tool sampling may have been reinforced by the occasional successful dips or may have been a form of response facilitation in reaction to other chimpanzees sampling their tools (Hoppitt et al., 2007).

\section{Termite Mound Design and Causal Understanding}

One limitation of our study relates to the physical properties of the artificial termite mound that we co-opted for this experiment, which may have impacted the chimpanzees' individual learning of the task. In the wild, and in our study, chimpanzees cannot see the reward inside the mounds. However, in the wild, chimpanzees create their own hole in the mound itself (with the first tool; Sanz \& Morgan, 2007); thus, they see the causal interplay between the use of the rigid tool, the piercing action, and the change in state of the termite mound (that a hole appears). In our study, unlike in the wild, tool insertion points were pre-drilled, and did not change in location across test sessions. In addition to this, the opacity of the artificial termite mound meant that the chimpanzees could not see if or when they had pierced the barrier (or indeed, that there was a barrier to pierce), which is in stark contrast to the outcome of piercing and puncturing actions in a wild setting. Thus, it may not have been so causally obvious that the chimpanzees needed to pierce a barrier to access the food. These combined factors might have resulted in a lack of environmental channeling of the correct order of tool use in each tested individual (or even the need for two tools). In other words, in the wild, it might make more sense (provide more positive feedback) to make a new hole first and then use a flexible tool, rather than work at a hole first made, but then deserted, by others. However, it may also be that chimpanzees might require much longer individual learning time to overcome their tendency to skip the rigid tool in their approach (from their previous years of experience using rigid wooden tools, this group of captive chimpanzees may be more likely to perseverate in using such tool forms than flexible ones - discussed further below). Future studies could test for the reoccurrence and stabilization of the correct sequence over an extended period of testing time.

\section{Prior Personal Experience and Conservatism}

Another factor to consider is the chimpanzees' prior experience with the task: it is possible that we inadvertently made the task too difficult by co-opting a task at which this group was already highly proficient. When chimpanzees are presented with a novel form of a familiar task, they can act conservatively (Hrubesch et al., 2009) and it may be that they are unable to adjust their strategies in response to a change in a (very) familiar task. Furthermore, not only did we require the chimpanzees to adopt a novel strategy to solve a familiar task, but the task changes were unknown to the chimpanzees (we changed the form of the tubes into which they had to dip, which were out of view of the chimpanzees). The only change visible to the chimpanzees were the novel tools we provided. Recent work has suggested that, when apes understand the causal demands of a task, they are not conservative, but can flexibly respond to changes in task design (Jacobson \& Hopper, 2019). Thus, the chimpanzees' extensive experience solving the task in one way (using one tool), with tubes that are considerably shorter, could have inhibited their ability to innovate a tool-set form, and yet we did not find this. Despite 
their prior experience with the task, and their lack of information about task changes, the chimpanzees did exhibit a novel approach to solving this modified task (i.e., the adoption of a tool-set form). Furthermore, for wild chimpanzees that use tool sets, it is proposed that they first learned to use one tool and then, perhaps via the ratchet effect, augmented the technique by using two tools in correct succession. In sum, this suggests that they are not conservative (sensu Hrubesch et al., 2009), but can adopt novel, more efficient strategies when needed (see also Jacobson \& Hopper, 2019).

In our study it is possible that the chimpanzees may have more consistently adopted tool-set use, however, if they had been provided with greater information and visual access about the causal mechanics of the task. Povinelli (2000) argued that "the principles of chimpanzee folk physics are founded upon things that can be directly perceived, including action sequences that can be generated from imagination or held as visual memory" (p. 307). In an example that is different, but not completely unrelated to the demands of our task, Povinelli tested chimpanzees on their ability to construct or deconstruct a tool to use on a particular apparatus (also see Price et al., 2009; Vale et al., 2016). In one version of Povinelli's task, the chimpanzees needed a straight tool (the "deconstructed" version) to reach the reward, and in another, they need a hook tool (the "constructed" version) to reach the reward. The chimpanzees in the study struggled to create the appropriate tool. More recently, Bania et al. (2009) replicated this experiment and found that the enculturated chimpanzees they worked with were highly successful at the same task. Bania and colleagues postulated that this was for several reasons, including the fact that the enculturated chimpanzees had extensive experience with tools during early development ( $0-3$ years of age). The chimpanzees Povinelli tested, on the other hand, began using tools after this critical developmental stage, similar to the chimpanzees we tested. This highlights the important interplay between personal experience, rearing history, and causal understanding when interpreting chimpanzees' tool use and problem-solving success. Related to this is the chimpanzees' ineffectual modifications of the flexible tools.

While the chimpanzees in our study could not modify the rigid tools that we provided, they could make modifications to the flexible, cardboard tools, and indeed they did so frequently. The majority of these modifications consisted of the chimpanzees fraying or shortening the tools. In the past, these same chimpanzees have been shown to modify stick tools for use in this termite mound in similar ways (Hopper, Tennie et al., 2015), suggesting that tool modification (including frayed, brushed tools) was already in their repertoire. Indeed, wild chimpanzees also typically modify tools in this manner (Bermejo \& Illera, 1999; Sanz et al., 2009). In the aforementioned replication of one of Povinelli's (2000) experiments, Bania et al. (2009) found that chimpanzees were more successful at the tool task when the required tool modification involved deconstructing a tool, rather than constructing one, and pointed out that wild chimpanzees typically modify tools by taking them apart, rather than assembling them (although see Shumaker et al., (2011) for examples of other kinds of ways in which chimpanzees have been reported to modify tools). In our study, and in contrast to the group's prior experience with tool modification (see Hopper, Tennie et al., 2015), shortening the tools quickly resulted in tools that were too short to reach the reward and so modification made the tools non-functional. Since the chimpanzees could not see the tubes, they did not have any way of assessing what was happening to their short tools (i.e., that they fell into the tubes and out of reach). The high rate of tool modifications (an average of nearly 50 modifications per 30-minute session) may potentially reflect perseveration on a previously successful, but now ineffectual, approach (Hopper, Tennie et al., 2015) or instead may be a mark of the chimpanzees' frustration. This is also in line with an increase in modifications in later sessions in tandem with a decreased engagement with the task more generally.

\section{Conclusion}

We provided zoo-housed chimpanzees with a novel task, two types of tools resembling wild tools in functionality, and a short period of time to demonstrate innovation of a correctly ordered two step toolset behavior. Chimpanzees in the wild perform this behavioral sequence, but it remains to be seen if this sequence is a culture-dependent trait, or a skill that falls within the ZLS of chimpanzees. The 
chimpanzees we tested did use the tools to extract rewards, and did use the tools in the required order, showing that the form of this tool set (its correct sequence) falls generally within the ZLS of chimpanzees. Thus, our methods not only allowed us to ask questions about chimpanzee learning and culture, but also to provide them with the opportunity to exhibit species-typical behavior through the provision of an enriching paradigm. However, our study was different from wild conditions in a number of ways and the correct sequence also did not solidify, (at least not in the time frame of our study). Future studies will be needed to determine what leads to a stabilizing of the behavioral sequence in the wild.

\section{Acknowledgments}

We thank Jennifer Vonk and Michael Beran for inviting us to participate in this special issue celebrating the $20^{\text {th }}$ anniversary of the publication of Povinelli's Folk Physics for Apes. This research was funded by the Leo S. Guthman Fund and, at the time of writing, LMH was supported by the Lincoln Park Zoo Women's Board. We would like to thank Maureen Leahy, Michael Brown-Palsgrove, and the animal care staff at the Regenstein Center for African Apes for their help and support of this project. We would also like to thank Callum Duffield for diligently coding all of the videos. CT received funding via the ERC STONECULT project during the writing of the manuscript. The project STONECULT has received funding from the European Research Council (ERC) under the European Union's Horizon 2020 research and innovation programme (grant agreement No. 714658).

\section{References}

Alem, S., Perry, C. J., Zhu, X., Loukola, O. J., Ingraham, T., Søvik, E., \& Chittka, L. (2016). Associative mechanisms allow for social learning and cultural transmission of string pulling in an insect. PLoS Biology, 14, e1002564.

Anonymous. (2005). So much more to know. Science 309, 78-102.

Bandini, E., Motes-Rodrigo, A., Steele, M. P., Rutz, C., \& Tennie, C. (2020). Examining the mechanisms underlying the acquisition of animal tool behaviour. Biology Letters, 16, 20200122.

Bandini, E., \& Tennie, C. (2017). Spontaneous reoccurrence of "scooping", a wild tool-use behaviour, in naïve chimpanzees. PeerJ, 5, e3814.

Bania, A. E., Harris, S., Kinsley, H. R., \& Boysen, S. T. (2009). Constructive and deconstructive tool modification by chimpanzees (Pan troglodytes). Animal Cognition, 12, 85-95.

Bates, D., Maechler, M., \& Bolker, B. (2012). lme4: Linear mixed-effects models using S4 classes [Computer software]. R package (version 0. 999999-0). http://CRAN.R-project.org/package1/4lme4

Bermejo, M., \& Illera, G. (1999). Tool-set for termite-fishing and honey extraction by wild chimpanzees in the Lossi Forest, Congo. Primates, 40, 619-627.

Biro, D., Inoue-Nakamura, N., Tonooka, R., Yamakoshi, G., Sousa, C., \& Matsuzawa, T. (2003). Cultural innovation and transmission of tool use in wild chimpanzees: Evidence from field experiments. Animal Cognition, 6, 213-223.

Boesch, C. (2020). Listening to the appeal from the wild. Animal Behavior and Cognition, 7, 257-263.

Boesch, C., Head, J., \& Robbins, M. M. (2009). Complex tool sets for honey extraction among chimpanzees in Loango National Park, Gabon. Journal of Human Evolution, 56, 560-569.

Bonnie, K., Ross, S. R., \& Lonsdorf, E. V. (2012). Flexibility and persistence of chimpanzee (Pan troglodytes) foraging behavior in a captive environment. American Journal of Primatology, 74, 661-668.

Boyd, R., \& Richerson, P. J. (2005). The origin and evolution of cultures. Oxford University Press.

Boyd, R., Richerson, P. J., \& Henrich, J. (2011). The cultural niche: Why social learning is essential for human adaptation. Proceedings of the National Academy of Sciences, 108, 10918-10925.

Brewer, S. M., \& McGrew, W. C. (1990). Chimpanzee use of a tool-set to get honey. Folia Primatologica, 54, 100104.

Calcutt, S. E., Lonsdorf, E. V., Bonnie, K. E., Milstein, M. S., \& Ross, S. R. (2014). Captive chimpanzees share diminishing resources. Behaviour, 151, 1967-1982.

Corat, C., Siqueira, J., \& Ottoni, E. B. (2016). Sequential organization and optimization of the nut-cracking behavior of semi-free tufted capuchin monkeys (Sapajus sp.) Primates, 57, 113-121. 
Dean, L. G., Kendal, R. L., Schapiro, S. J., Thierry, B., \& Laland, K. N. (2012). Identification of the social and cognitive processes underlying human cumulative culture. Science, 335, 1114-1118.

Deblauwe, I., Guislain, P., Dupain, J., \& van Elsacker, L. (2006). Use of a tool-set by Pan troglodytes troglodytes to obtain termites (Macrotermes) in the periphery of the Dja Biosphere Reserve, Southeast Cameroon. American Journal of Primatology, 68, 1191-1196.

Eschar, Y., Izar, P., Visalberghi, E., Resende, B., \& Fragaszy, D. (2016). When and where to practice: Social influences on the development of nut-cracking in bearded capuchins (Sapajus libidinosus). Animal Cognition, 19, 605-618.

Fay, J. M., \& Carroll, R. W. (1994). Chimpanzee tool use for honey and termite extraction in Central Africa. American Journal of Primatology, 34, 309-317.

Finestone, E., Bonnie, K. E., Hopper, L. M., Vreeman, V. M., Lonsdorf, E. V., \& Ross, S. R. (2014). The interplay between individual, social, and environmental influences on chimpanzee food choices. Behavioural Processes, 105, 71-77.

Fiore, A. M., Cronin, K. A., Ross, S. R. \& Hopper, L. M. (2020). Food cleaning by Japanese macaques: Innate, innovative, or cultural? Folia Primatologica, 91, 433-444.

Fragaszy, D., Izar, P., Visalberghi, E., Ottoni, E. B., \& de Oliveira, M. G. (2004). Wild capuchin monkeys (Cebus libidinosus) use anvils and stone pounding tools. American Journal of Primatology, 64, 359-366.

Hashimoto, C., Isaji, M., Koops, K., \& Furuichi, T. (2015). First records of tool-set use for ant-dipping by Eastern chimpanzees (Pan troglodytes schweinfurthii) in the Kalinzu Forest Reserve, Uganda. Primates, 56, 301305.

Helfman, G. S., \& Schultz, E. T. (1984). Social transmission of behavioural traditions in a coral reef fish. Animal Behaviour, 32, 379-384.

Hicks, T. C., Fouts, R. S., \& Fouts, D. H. (2005). Chimpanzee (Pan troglodytes troglodytes) tool use in the Ngotto forest, Central African Republic. American Journal of Primatology, 65, 221-237.

Hopper, L. M., Kurtycz, L. M., Ross, S. R., \& Bonnie, K. E. (2015). Captive chimpanzee foraging in a social setting: A test of problem solving, flexibility, and spatial discounting. PeerJ, 3, e833.

Hopper, L. M., Tennie, C., Ross, S. R., \& Lonsdorf, E. V. (2015). Chimpanzees create and modify probe tools functionally: A study with zoo-housed chimpanzees. American Journal of Primatology, 77, 162-170.

Hoppitt, W., Blackburn, L., \& Laland, K. N. (2007). Response facilitation in the domestic fowl. Animal Behaviour, 73, 229-238.

Horner, V., \& Whiten, A. (2005). Causal knowledge and imitation/emulation switching in chimpanzees (Pan troglodytes) and children (Homo sapiens). Animal Cognition, 8, 164-181.

Hrubesch, C., Preuschoft, S., \& van Schaik, C. (2009). Skill mastery inhibits adoption of observed alternative solutions among chimpanzees (Pan troglodytes). Animal Cognition, 12, 209-216.

Huffman, M. A., \& Hirata, S. (2004). An experimental study of leaf swallowing in captive chimpanzees: Insights into the origin of a self-medicative behavior and the role of social learning. Primates, 45, 113-118.

Humle, T. (2002). The tool repertoire of Bossou chimpanzees. In T. Matsuzawa, T. Humle, \& Y. Sugiyama (Eds.) The chimpanzees in Bossou and Nimba. (p. 61-71). Springer.

Jacobson, S. L. \& Hopper, L. M. (2019). Hardly habitual: Chimpanzees and gorillas show flexibility in their motor responses when presented with a causally-clear task. PeerJ, 7, e6195.

Kendal, R., Hopper, L. M., Whiten, A., Brosnan, S. F., Lambeth, S. P., Schapiro, S. J., \& Hoppitt, W. (2015). Chimpanzees copy dominant and knowledgeable individuals: Implications for cultural diversity. Evolution and Human Behavior, 36, 65-72.

Koops, K., Schöning, C., McGrew, W. C., \& Matsuzawa, T. (2015). Chimpanzees prey on army ants at Seringbara, Nimba Mountains, Guinea: Predation patterns and tool use characteristics. American Journal of Primatology, 77, 319-329.

Laland, K. N., \& Hoppitt, W. (2003). Do animals have culture? Evolutionary Anthropology: Issues, News, and Reviews: Issues, News, and Reviews, 12, 150-159.

Lonsdorf, E. V. (2006). What is the role of mothers in the acquisition of termite-fishing behaviors in wild chimpanzees (Pan troglodytes schweinfurthii)? Animal Cognition, 9, 36-46.

Lonsdorf, E. V., Ross, S. R., Linick, S. A., Milstein, M. S., \& Melber, T. N. (2009). An experimental, comparative investigation of tool use in chimpanzees and gorillas. Animal Behaviour, 77, 1119-1126.

Luncz, L. V., Sirianni, G., Mundry, R., \& Boesch, C. (2018). Costly culture: Differences in nut-cracking efficiency between wild chimpanzee groups. Animal Behaviour, 137, 63-73.

Marshall-Pescini, S., \& Whiten, A. (2008). Chimpanzees (Pan troglodytes) and the question of cumulative culture: An experimental approach. Animal Cognition, 11, 449-456. 
Matsuzawa, T., \& Yamakoshi, G. (1996). Comparison of chimpanzee material culture between Bossou and Nimba, West Africa. In A. E. Russon, K. A. Bard, \& S. T. Parker (Eds.), Reaching into thought: The minds of the great apes (p. 211-232). Cambridge University Press.

McGrew, W.C., \& Rogers, M.E. (1983). Brief report: Chimpanzees, tools, and termites: New record from Gabon. American Journal of Primatology, 5, 171-174.

McGrew, W. C. (1998). Culture in nonhuman primates? Annual Review of Anthropology, 27, 301-328.

McGrew, W. C., \& Marchant, L. F. (1999). Laterality of hand use pays off in foraging success for wild chimpanzees. Primates, 40, 509-513.

McGrew, W. C., \& Rogers, M. E. (1983). Brief report: Chimpanzees, tools, and termites: New record from Gabon. American Journal of Primatology, 5, 171-174.

Menzel, C., Fowler, A., Tennie, C., \& Call, J. (2013). Leaf surface roughness elicits leaf swallowing behavior in captive chimpanzees (Pan troglodytes) and bonobos (P. paniscus), but not in gorillas (Gorilla gorilla) or orangutans (Pongo abelii). International Journal of Primatology, 34, 533-553.

Mesoudi A., \& Thornton, A. (2018). What is cumulative cultural evolution? Proceedings of the Royal Society B, 285, 20180712.

Motes-Rodrigo, A., Majlesi, P., Pickering, T. R., Laska, M., Axelsen, H., Minchin, T. C., Tennie, C., \& HernandezAguilar, R.A. (2019). Chimpanzee extractive foraging with excavating tools: Experimental modeling of the origins of human technology. PloS One, 14, e0215644.

Muroyama, Y. (1991). Chimpanzees' choices of prey between two sympatric species of Macrotermes in the Campo Animal Reserve, Cameroon. Human Evolution, 6, 143-151.

Musgrave, S., Lonsdorf, E., Morgan, D., Prestipino, M., Bernstein-Kurtycz, L., Mundry, R., \& Sanz, C. (2020). Teaching varies with task complexity in wild chimpanzees. Proceedings of the National Academy of Sciences, 117, 969-976.

Musgrave, S., Morgan, D., Lonsdorf, E., Mundry, R., \& Sanz, C. (2016). Tool transfers are a form of teaching among chimpanzees. Scientific Reports, 6, 34783.

Neadle, D., Allritz, M., \& Tennie, C. (2017). Food cleaning in gorillas: Social learning is a possibility but not a necessity. PloS One, 12, e0188866.

Nishihara, T., Suzuki, S., \& Kuroda, S. (1995). Tool-set for termite-fishing by chimpanzees in the Ndoki Forest, Congo. Behaviour, 132, 219-235.

Pinker, S. (2010). The cognitive niche: Coevolution of intelligence, sociality, and language. Proceedings of the National Academy of Sciences, 107, 8993-8999.

Povinelli, D. (2000). Folk physics for apes: The chimpanzee's theory of how the world works. Oxford University Press.

Price, E. E., Lambeth, S. P., Schapiro, S. J., \& Whiten, A. (2009). A potent effect of observational learning on chimpanzee tool construction. Proceedings of the Royal Society B: Biological Sciences, 276, 3377-3383.

R Core Team (2019). R: A language and environment for statistical computing [Computer software]. R Foundation for Statistical Computing. https://www.R-project.org/

Reindl, E., Apperly, I. A., Beck, S. R., \& Tennie, C. (2017). Young children copy cumulative technological design in the absence of action information. Scientific Reports, 7, 1788.

Rendell, L., \& Whitehead, H. (2001). Culture in whales and dolphins. Behavioral and Brain Sciences, 24, 309-324.

Sanz, C. M., Call, J., \& Boesch, C. (2013). Tool use in animals: Cognition and ecology. Cambridge University Press.

Sanz, C., Call, J., \& Morgan, D. (2009). Design complexity in termite-fishing tools of chimpanzees (Pan troglodytes). Biology Letters, 5, 293-296.

Sanz, C. M., \& Morgan, D. B. (2007). Chimpanzee tool technology in the Goualougo Triangle, Republic of Congo. Journal of Human Evolution, 52, 420-433.

Sanz, C. M., \& Morgan, D. B. (2009). Flexible and persistent tool-using strategies in honey-gathering by wild chimpanzees. International Journal of Primatology, 30, 411-427.

Sanz, C., Morgan, D., \& Gulick, S. (2004). New insights into chimpanzees, tools, and termites from the Congo Basin. The American Naturalist, 164, 567-581.

Sanz, C. M., Schöning, C., \& Morgan, D. B. (2010). Chimpanzees prey on army ants with specialized tool set. American Journal of Primatology, 72, 17-24.

Sommer, V., Buba, U., Jesus, G., \& Pascual-Garrido, A. (2012). Till the last drop. Honey gathering in Nigerian chimpanzees. Ecotropica, 18, 55-64.

Shrout, P. E., \& Fleiss, J. L. (1979). Intraclass correlations: Uses in assessing rater reliability. Psychological Bulletin, 86, 420-428. 
Shumaker, R. W., Walkup, K. R., \& Beck, B. B. (2011). Animal tool behavior: The use and manufacture of tools by animals. Johns Hopkins University Press.

Tennie, C., Bandini, E., van Schaik, C. P., \& Hopper, L. M. (in press). The zone of latent solutions and its relevance to understanding ape cultures. Biology \& Philosophy.

Tennie, C., Call, J., \& Tomasello, M. (2009). Ratcheting up the ratchet: On the evolution of cumulative culture. Philosophical Transactions of the Royal Society of London B: Biological Sciences, 364, 2405-2415.

Tennie, C., Hopper, L. M., \& van Schaik, C. (2020). On the origin of cumulative culture: Consideration of the role of copying in culture-dependent traits and a reappraisal of the zone of latent solutions hypothesis. In L. M. Hopper \& S. R. Ross (Eds.), Chimpanzees in context. University of Chicago Press.

Tomasello, M. (1996). Do apes ape? In C. M. Heyes \& B. G. Galef, Jr. (Eds.), Social learning in animals: The roots of culture (pp. 319-346). Academic Press.

Tomasello, M., Kruger, A. C., \& Ratner, H. H. (1993). Cultural learning. Behavioral and Brain Sciences, 16, 495511.

van Schaik, C. P., Ancrenaz, M., Borgen, G., Galdikas, B., Knott, C. D., Singleton, I., Suzuki, A., Utami, S. S., \& Merrill, M. (2003). Orangutan cultures and the evolution of material culture. Science, 299, 102-105.

Vale, G. L., Flynn, E. G., Pender, L., Price, E., Whiten, A., Lambeth, S. P., Schapiro, S. J., \& Kendal, R. L. (2016). Robust retention and transfer of tool construction techniques in chimpanzees (Pan troglodytes). Journal of Comparative Psychology, 130, 24-35.

Weir, A. A., \& Kacelnik, A. (2006). A New Caledonian crow (Corvus moneduloides) creatively re-designs tools by bending or unbending aluminum strips. Animal Cognition, 9, 317-334.

Whiten, A., Goodall, J., McGrew, W. C., Nishida, T., Reynolds, V., Sugiyama, Y., Tutin, C. E. G., Wrangham, R. W., \& Boesch, C. (1999). Cultures in chimpanzees. Nature, 399, 682-685.

Whiten, A., McGuigan, N., Marshall-Pescini, S., \& Hopper, L. M. (2009). Emulation, imitation, over-imitation and the scope of culture for child and chimpanzee. Philosophical Transactions of the Royal Society of London B: Biological Sciences, 364, 2417-2428. 